the firm results are the proposed $\alpha$ carbon positions, and the correspondence of these to the known positions of ribonuclease $\mathrm{S}$ is convincingly demonstrated. It will be exciting to see the method applied to a completely new and unknown polypeptide or protein.

\section{New structure, new mechanism for trypsin}

\section{from a Correspondent}

IT may well be true that protein and enzyme crystallography has passed its zenith. But many laboratories engaged in this activity still continue to produce results that are often interesting, and even important. As the number of structures unravelled continues to increase, particular emphasis is being placed on structure-function relationships as well as on correlations between members of enzyme families.

The serine proteases are all enzymes involved in peptide bond cleavage during the processes of digestion, and the structures of two of them, chymotrypsin and elastase, were determined by $\mathrm{X}$-ray analysis several years ago. Stroud and his colleagues now report the details of their studies on inhibited complexes of a third such enzyme, trypsin. Inhibition of trypsin by diisopropylfuoro-phosphate proceeds irreversibly, to give a complex, DIP-trypsin, and Stroud, Kay and Dickerson (J. molec. Biol., 82, 185; 1974) have determined its three-dimensional structure, initially at low, and then at higher $(2.7 \AA)$ resolution. It is notable that the overall folding of the DIP-trypsin molecule is topologically very similar to that previously observed in chymotrypsin and elastase; the overall identity of sequence between these three is only $24 \%$. The active-site architectures are likewise closely related. DIP reacts uniquely with Ser 195 and the position of the $\gamma$-oxygen of this residue corresponds closely to that in acylchymotrypsin.

Trypsin is also specifically and reversibly inhibited by benzamidine, in a way that probably mimics arginine side chains in a specific substrate. Krieger, Kay and Stroud have also determined the structure of this complex, which they describe in an accompanying article (ibid., 209). The siting of the benzamidine in the enzyme-binding pocket, has induced alterations in the geometry of this pocket so that, in particular, it has undergone a marked closure so as to 'sandwich' the aromatic ring of the inhibitor molecule in between several peptide planes.

From the comparison at neutral $p \mathrm{H}$ of the structures of these two quite different inhibited derivatives of trypsin, Krieger et al. attempt to obtain information concerning the mechanism of the enzyme action. DIP-trypsin can be considered to be a model for the 'tetrahedral' intermediate widely accepted to be a covalent intermediate in the catalysis of serine proteases-whereas the benzamidine-trypsin complex may well represent a Michaelis complex (or indeed any other non-covalent intermediate, such as the often-suggested trans-conformation complex). Benzamidine is, in effect, a powerful competitive inhibitor for specific trypsin substrates, and it is quite reasonable to suppose that it binds in the same way as them. Moreover, the benzamidine-trypsin complex is stable at $p \mathrm{H} 8$, when the enzyme is totally in its active conformation. Any observed change in the protein structure may then be solely attributed to the actual binding of the pseudo-substrate, and not to any shift in the balance between active and inactive conformations. The structure of active free trypsin remains to be solved, although Stroud et al. believe that DIP-trypsin should resemble it quite closely.

Insertion of benzamidine into the binding pocket involves the formation of a salt bridge with the $\beta$-carboxyl group of Asp 189, this residue accounting for the unique specificity of trypsin among the serine proteases for positively charged substrates. It is striking that this bridge is formed without any movement of the carboxylic acid, this residue being constrained by the tertiary structure. Any other substrate whose basic side chain has not the required length, will then, in forming the salt bridge, move the susceptible bond of the substrate away from its optimal catalysis position. The binding of benzamidine effectively induces certain changes in the active site, which in all favour rapid subsequent hydrolysis in the case of the specific substrates. The readjustment of the position of the most important residues of the enzyme when it binds a positively-charged substrate, may also explain the activating power of small amines for the hydrolysis of non-specific substrates.

It is worth noting that the chargerelay transfer mechanism involving the catalytic triad $\mathrm{Asp}_{102}, \mathrm{His}_{57}$ and $\mathrm{Ser}_{195}$ proposed by Blow et al. (Nature, 221, $337 ; 1969)$, for the acylation and deacylation of chymotrypsin, can also be applied to trypsin. Stroud et al. make use of the recent results obtained with $\alpha$-lytic protease (an elastase-like serine protease), by Richards and his colleagues (J. biol. Chem., 248, 8306; 1973), using ${ }^{13} \mathrm{C}$ magnetic resonance studies with selective labellings in suggesting that the proton accepter is the aspartic acid, and not the histidine residue. The imidazole ring of this histidine, which remains neutral throughout catalysis, serves simply as a relay between the serine and the aspartic acid residues. It thus keeps the carboxylate in a hydrophobic environment such that its $p \mathrm{~K}$ is increased to 6.7 . The advantage of this new scheme (which is at variance with hitherto widely accepted doctrine), is that it implies no charge separation during the formation of the tetrahedral intermediate, and is therefore on the face of it, to be preferred energetically.

\section{Limited progress with quantum gravity}

from a Correspondent

Most people would regard quantum theory and gravitation theory as two quite disjoint subjects; the first concerned with microscopic systems such as atoms, the second with objects of astronomical dimensions. Nevertheless, the marriage of these two unlikely bedfellows has fascinated a generation of physicists, for although quantum gravitional effects are deemed to be forever beyond the scope of direct experimental verification, the aesthetic appeal of a theoretical unification of these disciplines has always been compelling.

One of the driving forces behind research into quantum gravity is J. Wheeler (Princeton University), who has been visiting Britain during the past few weeks. His visit coincided with a conference on the subject held at the Rutherford Laboratory on February 15 and 16 , and organised by D. Sciama (University of Oxford). The large number of participants testified to the extraordinary degrce of interest in Britain for what is beginning to seem a theoretical obsession. With the sort of optimistic statements made recently by workers active in the subject, the conference might have been expected to produce convincing evidence of definite progress. In fact, it succeeded in gencrating a deep scepticism.

C. Isham (King's College, London) began with a review of the various levels of theoretical sophistication available to the would-be gravity quantiser, each level having its particular brand of ambiguities and problems. The presentation of technical results by $M$. Duff (University of Oxford) only served to confirm the extraordinary lengths which are neeessary in order to make even minor progress. Pessimism mounted with evidence supplied by J. Taylor (King's College) that if gravitation is to be described by general relativity, then apparently it would be unrenormalisable in the usual sense familiar from quantum electrodynamics. That is, not only do infinite quantities appear everywhere in the theory, but they appear an infinite number of times for even the simplest type of process. Quantum gravity, said Taylor, "is dead". 